\title{
Measurement properties of depression questionnaires in patients with diabetes: a systematic review
}

\author{
Susan E. M. van Dijk ${ }^{1}$ Marcel C. Adriaanse ${ }^{1}$. Lennart van der Zwaan ${ }^{1}$. Judith E. Bosmans ${ }^{1}$. \\ Harm W. J. van Marwijk ${ }^{2,3} \cdot$ Maurits W. van Tulder ${ }^{1} \cdot$ Caroline B. Terwee $^{4}$
}

Accepted: 5 January 2018 / Published online: 2 February 2018

(c) The Author(s) 2018. This article is an open access publication

\begin{abstract}
Purpose To conduct a systematic review on measurement properties of questionnaires measuring depressive symptoms in adult patients with type 1 or type 2 diabetes.

Methods A systematic review of the literature in MEDLINE, EMbase and PsycINFO was performed. Full text, original articles, published in any language up to October 2016 were included. Eligibility for inclusion was independently assessed by three reviewers who worked in pairs. Methodological quality of the studies was evaluated by two independent reviewers using the COnsensus-based Standards for the selection of health Measurement INstruments (COSMIN) checklist. Quality of the questionnaires was rated per measurement property, based on the number and quality of the included studies and the reported results.

Results Of 6286 unique hits, 21 studies met our criteria evaluating nine different questionnaires in multiple settings and languages. The methodological quality of the included studies was variable for the different measurement properties: $9 / 15$ studies scored 'good' or 'excellent' on internal consistency, 2/5 on reliability, 0/1 on content validity, 10/10 on structural validity, $8 / 11$ on hypothesis testing, $1 / 5$ on cross-cultural validity, and 4/9 on criterion validity. For the CES-D, there was strong evidence for good internal consistency, structural validity, and construct validity; moderate evidence for good criterion validity; and limited evidence for good cross-cultural validity. The PHQ-9 and WHO-5 also performed well on several measurement properties. However, the evidence for structural validity of the PHQ-9 was inconclusive. The WHO-5 was less extensively researched and originally not developed to measure depression.
\end{abstract}

Conclusion Currently, the CES-D is best supported for measuring depressive symptoms in diabetes patients.

Keywords Diabetes $\cdot$ Depression questionnaires $\cdot$ Measurement properties $\cdot$ COSMIN checklist

Electronic supplementary material The online version of this article (https://doi.org/10.1007/s11136-018-1782-y) contains supplementary material, which is available to authorized users.

Marcel C. Adriaanse

marcel.adriaanse@vu.nl

1 Department of Health Sciences, Faculty of Earth and Life Sciences, Amsterdam Public Health Research Institute, VU University Amsterdam, Amsterdam, The Netherlands

2 Department of General Practice and Elderly Medicine and the Amsterdam Public Health Research Institute, VU University Medical Center, Amsterdam, The Netherlands

3 Manchester Academic Health Sciences Centre and NIHR School for Primary Care Research, The University of Manchester, Manchester, UK

4 Department of Epidemiology and Biostatistics and the Amsterdam Public Health Research Institute, VU University Medical Center, Amsterdam, The Netherlands

\section{Introduction}

Diabetes is a common and serious chronic disease that is estimated to affect more than 350 million people worldwide [1]. Adult patients with diabetes type 1 or type 2 often have comorbid depression. Up to $20 \%$ of diabetes patients have major depressive disorder and up to $40 \%$ have clinically relevant depressive symptoms at one point in time according to the criteria of the Diagnostic and Statistical Manual of Mental Disorders-IV (DSM-IV) [2-4].

Comorbid depression in patients with diabetes is associated with poorer adherence to medical treatment and more difficulties complying with self-care instructions compared to patients with diabetes alone [5]. These patients also experience adverse health outcomes, such as poorer glycemic control [6], more diabetes complications [7], lower quality of 
life [8] and higher risk of morbidity and all-cause mortality. Furthermore, they use more healthcare resources resulting in higher healthcare costs [9].

Given the high prevalence of comorbid depression and associated adverse health outcomes, it is important to monitor depressive symptoms in diabetes patients on a regular basis, for example to evaluate changes during and after an intervention. Clinical guidelines recommend doing this with standardized questionnaires [10]. This way, depression treatment can be optimized and adjusted when necessary [11].

A wide variety of questionnaires is available to measure depressive symptoms. Questionnaires that are frequently used in diabetic populations are the Center of Epidemiological Studies-Depression Scale (CES-D) [12], the Hospital Anxiety and Depression Scale-Depression (HADS-D) [13], the Patient Health Questionnaire-9 (PHQ-9) [14] and the Beck Depression Inventory-II (BDI-II) [15]. However, these questionnaires generally assess symptoms of depression that may overlap with common symptoms of a medical illness such as diabetes (e.g., fatigue, changes in weight and appetite). Although many of these questionnaires have undergone extensive psychometric testing, an overview of their performance in this specific diabetes population is lacking [16]. This information is valuable because measurement properties may vary across populations. Also, a large number of questionnaires is available, while no recommendations are available which one to use to monitor depressive symptoms in diabetes patients. This makes it difficult to select the most suitable questionnaire for monitoring and evaluating depressive symptoms in diabetes patients.

Systematic, comparative evidence on the measurement properties of these questionnaires, used for evaluating depressive symptoms within patients on a continuous scale, is required by physicians and researchers.

Measurement properties are divided in three domains: reliability, validity, and responsiveness [17]. A reliable questionnaire performs its measurements precisely, without too much measurement error. A valid questionnaire has the ability to measure the intended construct (and not something else). A responsive questionnaire is sensitive to changes in the construct to be measured [17]. Next to these three domains, it is important that a measurement instrument is interpretable, meaning that the quantitative results of the questionnaire can be translated to clinically meaningful conclusions [17].

Roy et al., conducted a comprehensive review in 2012 in which they identified frequently used depression questionnaires used in diabetes patients. They conclude that the BDI, PHQ-9, CES-D and the HADS-D are most used. However, they did not systematically evaluate the measurement properties of the included questionnaires. It is therefore not known which questionnaire is most reliable and valid for measuring depressive symptoms in diabetes patients. Therefore, the aim of this study was to summarize the comprehensive research on the measurement properties (reliability, validity and responsiveness) of questionnaires used to evaluate depressive symptoms in adult patients with type 1 and type 2 diabetes. Knowledge generated from this study may help clinicians and researchers to make a better evidenced-based selection of questionnaires for the evaluation of depressive symptoms among diabetes patients.

\section{Methods}

\section{Design}

A systematic review of the literature was conducted according to the recommendations from the COnsensus-based Standards for the selection of health status Measurement INstruments (COSMIN) initiative (https://www.cosmin.nl). According to these recommendations, the literature was systematically searched; the quality of the included studies was assessed; the results of the studies were rated against predefined criteria; the results of multiple studies per measurement property were systematically synthesized, and levels of evidence were applied. A detailed description of the used methods is provided below.

\section{Data sources, search strategy, and study selection}

We searched MEDLINE, EMBASE, and PSYCINFO from inception [i.e. with no specified beginning date up until (and including)] to October 2016. The investigators developed the search after consulting an information specialist (a university librarian). The search strategy consisted of search terms for depression and type 1 and type 2 diabetes. Different from the COSMIN recommendations, terms regarding type of instrument and measurement properties (reliability, validity and responsiveness) were not used, because we wanted to reduce the chance of missing any relevant articles. The used search terms are shown in S1 Appendix.

We included all studies published in any language on the measurement properties of self-report questionnaires measuring depressive symptoms (as defined as such by the authors of the paper) in type 1 or type 2 diabetes patients (i.e. at least $80 \%$ of the study population had diabetes). Studies were included in the review when the questionnaire under study was used to measure depressive symptoms, even if the questionnaire was not originally developed for this purpose. Only studies that reported measurement properties of these questionnaires, i.e. reliability, validity and/or responsiveness, were included in the review. Studies that only assessed the diagnostic accuracy of a questionnaire were not included, since these studies are concerned with the ability 
of a questionnaire to detect a target condition, while in this review, the focus is on the evaluative use of questionnaires to monitor the severity of depressive symptoms over time.

Three reviewers (SD, LZ, MA) independently assessed the titles and abstracts of the retrieved studies to identify relevant studies. The reviewers worked in pairs and discussed their selection. When both reviewers agreed a study was possibly relevant or when consensus was not reached, the full text article was retrieved and read by all three authors to determine whether in- and exclusion criteria were met. For the final inclusion of an article, after reading of the full text, consensus between all three reviewers (SD, LZ and MA) was needed. When consensus was not reached, a fourth reviewer decided (CT). The reference lists of the included articles were checked by two reviewers independently of each other (SD and LZ) and related citations of relevant articles found in MEDLINE were screened to identify additional relevant studies.

\section{Identification of studied measurement properties}

Two reviewers (SD and LZ) independently identified for each study which measurement properties were reported. When no consensus was reached, a third reviewer discussed the interpretation of the reviewers with them and decided based on her leading expertise in the field of measurement properties (CT). Based on the COSMIN recommendations, three domains of measurement properties were distinguished: reliability, validity and responsiveness [17].

\section{Reliability}

A self-reported health questionnaire is considered reliable when it (a) is internally consistent, with all items (in a subscale) showing a high degree of interrelatedness (Cronbach's $\alpha$.70-.90); (b) has high reliability, which means that a high proportion of the variability in the measurement outcome is caused by real differences between or changes within patients; and (c) does not introduce a lot of measurement error (differences in the measurement outcome that cannot be attributed to differences in the construct to be measured [17]).

\section{Validity}

Validity of a questionnaire includes (a) content validity, or how well a questionnaire reflects the construct it is supposed to measure; (b) construct validity, or to which degree the measurement outcome reflects the dimensional structure of a questionnaire (structural validity), the degree to which the scores of a questionnaire are consistent with hypotheses based on theoretical knowledge of the construct to be measured (hypothesis testing) and the degree to which a translated questionnaire performs similarly to the original version (cross-cultural validity); and (c) criterion validity, or how well the outcome of a questionnaire reflects the outcome of a 'gold standard' to measure the same construct [17].

\section{Responsiveness}

A questionnaire is considered responsive when it is able to detect change in the construct to be measured.

Next to these three domains of measurement properties, it is important that the results of a questionnaire are interpretable [17].

\section{Assessment of the methodological quality of the included studies}

After consensus was reached on which properties were assessed in the selected studies, the methodological quality of the assessment of each studied measurement property was rated for all studies using the COSMIN checklist [18]. This checklist consists of 9 boxes that correspond with the defined measurement properties. In each box, methodological standards are presented on how each measurement property should be assessed. The 9 boxes consist of 5 (content validity) through 18 (hypothesis testing) items. These items are scored in a standardized way on a 4-point scale (i.e. "poor", "fair", "good" or "excellent") [19]. An overall score of the methodological quality for each box was determined by taking the lowest rating of any of the items in that box, since a low rating on any of the items signals a significant risk of bias. The quality assessment was independently done by three reviewers who worked in pairs (SD and LZ; SD and $\mathrm{MA})$. These reviewers were trained by one of the developers of the COSMIN checklist (CT). A third reviewer (CT) decided when consensus on any item was not reached.

\section{Data extraction}

Characteristics of the study design and questionnaires were extracted and summarized for all included studies. Study design characteristics included questionnaire used, sample size, mean age, gender distribution, proportion of diabetes patients in the sample, country and setting in which the study was performed and language version of the used questionnaire. The following questionnaire characteristics were extracted: construct aimed to be measured, target population, number of items, subscales of the questionnaire, score range of the items and total scores, usual cut-points for depression, administration time and recall period.

The results regarding the reported measurement properties were extracted by two reviewers (SD and LZ), independently. Results on the instrument quality were abstracted for 
every measurement property separately using a standardized data extraction form. We used common criteria to classify results as positive (good measurement quality of the questionnaire), inconclusive or negative (poor measurement quality of the questionnaire) [20]. The used criteria for quality of measurement properties can be viewed in S2 Appendix.

\section{Data synthesis}

To rate the overall quality of the questionnaires, we combined the results on each measurement property with the ratings of methodological quality in each box, the number of studies in which the measurement property was investigated and the consistency of the results. In the data synthesis, only results of studies of excellent, good or fair methodological quality are considered, as recommended by the COSMIN initiative. An overall 'level of evidence' per measurement property was assigned to each individual questionnaire in accordance with previously performed systematic literature reviews $[21,22]$. As a result of this process, measurement properties were rated as positive, inconclusive or negative, with strong, moderate, limited or unknown level of evidence. In Table 1, the criteria used in this rating system are further explained. Although questionnaires are often evaluated using different language versions and their measurement properties are not necessarily similar across countries, results were summarized for every questionnaire, regardless of language version because there were not enough data to study differences in measurement properties between language versions. The data synthesis was independently performed by two reviewers (SD and MT) and in case consensus was not reached, a third reviewer (CT) made a final decision taking the arguments of the other reviewers into account.

\section{Results}

\section{Search strategy, inclusion of relevant studies and studied questionnaires}

The search strategy yielded 6286 unique articles, of which 63 were selected based on title and abstract. After reading the full text version, 21 were eligible for inclusion. Searching related citations in MEDLINE and reference lists of included papers yielded no additional articles. Thus, in total, 21 relevant studies were included in this review [23-43]. The inclusion process is described in more detail in Fig. 1.

Nine different questionnaires were evaluated: the CES-D [12], the Chinese version of the Clinically Useful Depression Outcome Scale (CUDOS) [44], the Depressive Cognition Scale (DCS) [45], the Depression in Diabetes Self-Rating Scale (DDSRS) [27], the Edinburgh Depression Scale [46], the HADS-D [13], the McSad [47], the PHQ-9 [14] and the 5-item World Health Organisation Well Being Index (WHO5) [48]. Information regarding the selected articles and the depression questionnaires is presented in Tables 2 and 3.

Sample sizes of the included studies varied widely, ranging from 24 [30] to 1656 [24]. The population in which the questionnaires were assessed differs greatly regarding age, languages and settings. For example, mean age of the participants ranged from 29 [34] to 82 [39]. The questionnaires were assessed in ten different languages (Japanese [23], Dutch [24, 25, 28, 31, 43], Chinese [26, 32, 37, 40, 41], Polish [27], Turkish [29], Sylheti [30], Mirpuri [30], English [33, 36-39, 42], Portuguese [34, 35] and Spanish [36]). The setting in which the questionnaires were researched differed between studies (for example, primary care, hospital outpatient clinics, university hospitals and patient support group organizations). Most samples only consisted of type 2 diabetes patients, but three studies also included type 1 diabetes patients [23, 25, 37]. Four studies did not specify the number of type 1 and type 2 diabetics [31, 34, 35, 39].
Table 1 Criteria for assigning a level of evidence rating

\begin{tabular}{lll}
\hline Level of evidence & Rating & Criteria \\
\hline Strong & +++ or --- & $\begin{array}{l}\text { Consistent findings in multiple studies of good } \\
\text { methodological quality, or in one study of excellent } \\
\text { methodological quality }\end{array}$ \\
Moderate & ++ or -- & $\begin{array}{l}\text { Consistent findings in multiple studies of fair methodo- } \\
\text { logical quality, or in one study of good methodologi- } \\
\text { cal quality }\end{array}$ \\
Limited & + or - & $\begin{array}{l}\text { Evidence from one study of fair methodological quality } \\
\text { Inconclusive evidence }\end{array}$ \\
Unknown & $+/-$ & Only studies of poor methodological quality \\
\hline
\end{tabular}

Criteria were based on previously performed systematic reviews [21, 22] 


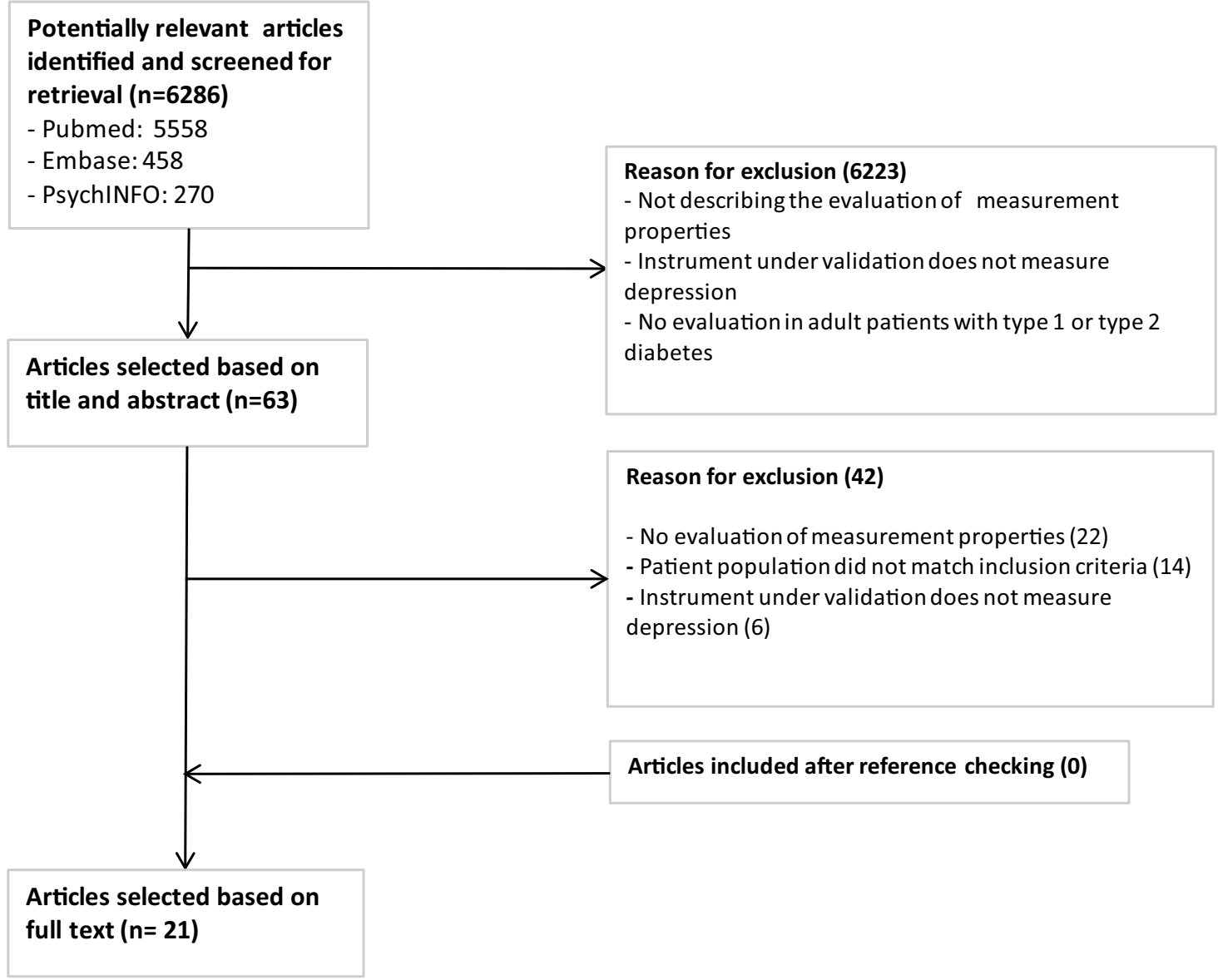

Fig. 1 Selection of studies flowchart

\section{Methodological quality}

The methodological quality of the studies was variable ranging from 'poor' to 'excellent' (Table 4). Structural validity was rated as 'good' and 'excellent' for all studies. The most frequently assessed measurement properties were internal consistency (17 studies [23, 25-29, 32-41, 43]) and hypothesis testing (13 studies [23, 25-29, 31-33, 35, 36, 38, 39]). Only few studies examined reliability (5 studies) [26, 28, $33,40,41]$, cross-cultural validity (5 studies) [23, 26, 30, $32,34]$ and content validity (1 study) [30]. There were no studies that examined measurement error, responsiveness or interpretability.

Two out of 15 studies scored 'excellent' [25, 33] and seven studies scored 'good' [23, 26, 29, 35, 36, 38, 39] on internal consistency, Lower quality ratings were mostly caused by not assessing or describing the dimensionality of a questionnaire and not assessing internal consistency for every subscale of a questionnaire separately [27, 28, 32, $37,40,41,43]$, or having a small or not representative study population $[32,34]$.
Five studies assessed the reliability of the investigated questionnaire, of which one study was rated 'excellent' [33] and one study was rated 'good' [26]; the other three studies were of poor methodological quality [28, 40, 41] due to flaws in the study design or statistical methods used.

One study [30] reported content validity and was rated 'poor' due to methodological flaws in the design of the study. Of the ten studies reporting structural validity [23-26, 29, 33, 35, 36, 41], two were rated 'excellent' [25, 33 ] and the other eight studies were rated 'good'. The difference between a 'good' and 'excellent' rating was caused by differences in reporting on missing values and drop-out in the study, or differences in sample size, with excellent studies having a larger sample.

One out of 11 studies reporting hypothesis testing was rated 'excellent' [31]. Seven studies were rated 'good' [25, $29,33,35,36,38,39]$. The main reasons for a lower quality score were small sample size $[28,32]$ or not sufficiently specifying prior hypotheses [23, 26, 27].

Of the five studies assessing cross-cultural validity, one study was rated 'good' [34]. Lower quality scores were mainly caused by flaws in the translation process, 
Table 2 Characteristics of the included studies

\begin{tabular}{|c|c|c|c|c|c|c|c|c|}
\hline $\begin{array}{l}\text { Studies in } \\
\text { alphabetic } \\
\text { order }\end{array}$ & Instruments & Sample size & $\begin{array}{l}\text { Mean age in } \\
\text { years (SD) }\end{array}$ & Male $(\%)$ & $\begin{array}{l}\text { DM1/DM2 } \\
\text { (\% of total } \\
\text { sample) }\end{array}$ & $\begin{array}{l}\text { Country in } \\
\text { which study } \\
\text { was performed }\end{array}$ & Setting & Language \\
\hline $\begin{array}{c}\text { Awata et al. } \\
\text { [23] }\end{array}$ & WHO-5 & $\begin{array}{l}129 \\
65 \text { (criterion } \\
\quad \text { validity) }\end{array}$ & $54(10)$ & 55 & $16 / 84$ & Japan & $\begin{array}{c}\text { University } \\
\text { hospital }\end{array}$ & Japanese \\
\hline $\begin{array}{c}\text { Carter et al. } \\
\text { [42] }\end{array}$ & CES-D & 305 & $56.9(11.1)$ & 45 & $1 / 100$ & Canada & $\begin{array}{l}\text { Rehabilitation } \\
\text { institute }\end{array}$ & English \\
\hline $\begin{array}{l}\text { de Cock et al. } \\
\text { [24] }\end{array}$ & EDS & 1656 & $\begin{array}{l}65 / 67 \\
(10 / 10.6)^{\mathrm{a}}\end{array}$ & 50 & $0 / 100$ & $\begin{array}{l}\text { The Nether- } \\
\text { lands }\end{array}$ & Primary care & Dutch \\
\hline $\begin{array}{l}\text { Hajos et al. } \\
\text { [25] }\end{array}$ & WHO-5 & 933 & 53.4 & 49 & $41 / 59$ & $\begin{array}{l}\text { The Nether- } \\
\text { lands }\end{array}$ & $\begin{array}{l}\text { Hospital } \\
\text { outpatient } \\
\text { clinic }\end{array}$ & Dutch \\
\hline Hsu et al. [26] & CUDOS & 214 & $62.6(13.2)$ & 45 & $0 / 100$ & Taiwan & $\begin{array}{l}\text { University } \\
\text { hospital } \\
\text { outpatient } \\
\text { clinics }\end{array}$ & Chinese \\
\hline $\begin{array}{c}\text { Janssen et al. } \\
\text { [43] }\end{array}$ & PHQ-9 & 793 & $62.4(7.7)$ & 67 & $0 / 100$ & $\begin{array}{l}\text { The Nether- } \\
\text { lands }\end{array}$ & $\begin{array}{l}\text { Community- } \\
\text { based } \\
\text { sample }\end{array}$ & Dutch \\
\hline Kokoszka [27] & DDSRS & 101 & $63(11)$ & 50 & $0 / 100$ & Poland & $\begin{array}{l}\text { Medical Uni- } \\
\text { versity }\end{array}$ & Polish \\
\hline $\begin{array}{l}\text { Lamers et al. } \\
\text { [28] }\end{array}$ & PHQ-9 & $\begin{array}{l}365 \text { (internal } \\
\text { consistency, } \\
\text { criterion } \\
\text { validity) } \\
226 \text { (hypoth- } \\
\text { esis testing) } \\
27 \text { (reliability) }\end{array}$ & $71(6.9)^{\mathrm{b}}$ & $52^{\mathrm{b}}$ & $0 / 100$ & $\begin{array}{l}\text { The Nether- } \\
\text { lands }\end{array}$ & Primary care & Dutch \\
\hline $\begin{array}{l}\text { Lehman et al. } \\
\text { [29] }\end{array}$ & CES-D & 151 & $56(10)$ & 46 & $0 / 100$ & Turkey & $\begin{array}{l}\text { University } \\
\text { hospital } \\
\text { outpatient } \\
\text { clinics }\end{array}$ & Turkish \\
\hline $\begin{array}{l}\text { Lloyd et al. } \\
\text { [30] }\end{array}$ & $\begin{array}{l}\text { PHQ-9, } \\
\text { WHO-5 }\end{array}$ & 24 & 55 & 50 & $0 / 100$ & $\begin{array}{c}\text { UK (Bangla- } \\
\text { deshi and } \\
\text { Pakistani) }\end{array}$ & $\begin{array}{l}\text { Hospital } \\
\text { outpatient } \\
\text { clinic }\end{array}$ & Sylheti, Mirpuri \\
\hline $\begin{array}{l}\text { Papageorgiou } \\
\text { et al. [31] }\end{array}$ & McSad & 114 & $44(14.1)$ & 22 & $? / ?^{\mathrm{c}}$ & $\begin{array}{l}\text { The Nether- } \\
\text { lands }\end{array}$ & $\begin{array}{l}\text { Members of } \\
\text { a diabetes } \\
\text { patient } \\
\text { organisation }\end{array}$ & Dutch \\
\hline $\begin{array}{l}\text { Rankin et al. } \\
\text { [32] }\end{array}$ & CES-D & 30 & $\begin{array}{l}\text { range } 46 \text { thru } \\
\quad 80\end{array}$ & 57 & $0 / 100$ & United States & $\begin{array}{l}\text { Comprehen- } \\
\text { sive health } \\
\text { care centre }\end{array}$ & Chinese \\
\hline $\begin{array}{l}\text { Reddy et al. } \\
\text { [33] }\end{array}$ & $\begin{array}{l}\text { PHQ-9, } \\
\text { HADS-D }\end{array}$ & $\begin{array}{l}462 \text { (PHQ- } \\
\text { 9)/561 } \\
\text { (HADS-D) }\end{array}$ & 70 & 55 & $0 / 100$ & Australia & Primary care & English \\
\hline $\begin{array}{l}\text { Sousa et al. } \\
\text { [34] }\end{array}$ & DCS & 40 & $29.25(10.23)$ & 30 & $? / ?^{\mathrm{c}}$ & Brazil & $\begin{array}{l}\text { Convenience } \\
\text { sample }\end{array}$ & Portuguese \\
\hline $\begin{array}{l}\text { Sousa et al. } \\
\text { [35] }\end{array}$ & DCS & 82 & $61.28(11.37)$ & 35 & $? / ?^{\mathrm{c}}$ & Brazil & Primary care & Portuguese \\
\hline $\begin{array}{l}\text { Stahl et al. } \\
\text { [37] }\end{array}$ & CES-D & $\begin{array}{l}522 \text { (internal } \\
\text { consistency) } \\
291 \text { (criterion } \\
\text { validity) }\end{array}$ & $55(13)$ & - & $3.5 / 96.5$ & USA & $\begin{array}{l}\text { Hospital dia- } \\
\text { betes centre }\end{array}$ & $\begin{array}{l}\text { Chinese, Malay, } \\
\text { Indian }\end{array}$ \\
\hline $\begin{array}{l}\text { Sultan and } \\
\text { Fisher [36] }\end{array}$ & CES-D & 502 & $53.6(8.8)$ & 54 & $0 / 100$ & USA & $\begin{array}{l}\text { Community } \\
\text { based sam- } \\
\text { ple }\end{array}$ & $\begin{array}{l}\text { English, Spa- } \\
\text { nisch }\end{array}$ \\
\hline $\begin{array}{l}\text { Zauszniewski } \\
\text { et al. [38] }\end{array}$ & CES-D & 80 & 82 & 30 & $0 / 100$ & USA & Hospital & English \\
\hline
\end{tabular}


Table 2 (continued)

\begin{tabular}{|c|c|c|c|c|c|c|c|c|}
\hline $\begin{array}{l}\text { Studies in } \\
\text { alphabetic } \\
\text { order }\end{array}$ & Instruments & Sample size & $\begin{array}{l}\text { Mean age in } \\
\text { years (SD) }\end{array}$ & Male $(\%)$ & $\begin{array}{l}\text { DM1/DM2 } \\
\text { (\% of total } \\
\text { sample) }\end{array}$ & $\begin{array}{l}\text { Country in } \\
\text { which study } \\
\text { was performed }\end{array}$ & Setting & Language \\
\hline $\begin{array}{l}\text { Zauszniewski } \\
\text { and Graham } \\
\text { [39] }\end{array}$ & DCS & 83 & 46 & 0 & $? / ?^{\mathrm{c}}$ & USA & Hospital & English \\
\hline $\begin{array}{l}\text { Zhang et al. } \\
\text { [40] }\end{array}$ & PHQ-9 & $\begin{array}{c}586 \text { (internal } \\
\text { consistency) } \\
40 \text { (reliability) } \\
99 \text { (criterion } \\
\text { validity) }\end{array}$ & $55.1(9.5)$ & 59 & $0 / 100$ & China & $\begin{array}{l}\text { Hospital } \\
\text { outpatient } \\
\text { clinic }\end{array}$ & Chinese \\
\hline $\begin{array}{l}\text { Zhang et al. } \\
\text { [41] }\end{array}$ & CES-D & $\begin{array}{l}545 \text { (internal } \\
\text { consistency, } \\
\text { structural } \\
\text { validity) } \\
40 \text { (reliability) } \\
97 \text { (criterion } \\
\text { validity) }\end{array}$ & $54.6(9.5)$ & 59 & $0 / 100$ & China & $\begin{array}{l}\text { Hospital } \\
\text { outpatient } \\
\text { clinic }\end{array}$ & Chinese \\
\hline
\end{tabular}

CES-D Centre for Epidemiological Studies Depression Scale, CUDOS Clinically Useful Depression Outcome Scale, DCS Depression Cognition Scale, DDSRS Depression in Diabetes Self-Rating Scale, DM1 diabetes mellitus type 1, DM2 diabetes mellitus type 2, EDS Edinburgh Depression Scale, HADS-D Hospital Anxiety and Depression Scale-depression, PHQ-9 Patient Health Questionnaire-9, SD standard deviation, UK United Kingdom, USA United States of America, WHO-5 World Health Organization-Five Well-Being Index

${ }^{a}$ Mean and standard deviation reported separately for male/female participants

${ }^{\mathrm{b}}$ Characteristics of the total cohort in the study (not only diabetes patients) $(N=713)$

${ }^{\mathrm{c}}$ No details were reported on the number of type 1 and type 2 diabetes patients. However, the total sample consisted of $100 \%$ diabetes patients (either type 1 or type 2 )

not testing the translation in patients with diabetes before using the questionnaire in this population $[23,26,32]$ and small sample size [30].

Nine studies assessed criterion validity; four of these studies scored 'good' [28, 40, 41, 43]. The main reasons for a poor or fair rating were not using an accurate reference standard for measuring depression [25, 27], flaws in the study design $[23,27]$ or using a case control design without correction [23], thereby inflating estimates of criterion validity [49]. None of the studies were rated excellent because no gold standard exists to measure depression.

\section{Measurement properties of questionnaires measuring depressive symptoms}

Table 5 summarizes all results on measurement properties for each questionnaire. The final judgment on the level of evidence for the quality of the questionnaires per measurement property is presented in Table 6 . Since none of the studies assessed measurement error, responsiveness or interpretability, these properties are not included in the tables.

\section{CES-D}

The CES-D was assessed in six different languages in six studies [29, 32, 36, 37, 39, 41]. For internal consistency, structural validity, hypothesis testing, cross-cultural validity and criterion validity, there is predominantly strong to moderate evidence for good performance of the CES-D. Although not all studies assessing structural validity found the same factor structure, the two dominant factors (positive affect and negative affect) were found in every study [29, 36, 41, 42]. The additional factors found by Zhang et al. [41] and Carter et al. [42] all correlate highly with the negative affect factor. Therefore, we consider the evidence on structural validity consistent. One study evaluated reliability, but was of poor methodological quality [41]. Therefore, it was not possible to draw conclusions about the reliability of the CES-D.

\section{CUDOS}

The measurement properties of the CUDOS-Chinese were assessed in one study [28]. Results for internal consistency, reliability, structural validity, hypothesis testing and criterion validity were available. For internal consistency, inconsistent findings on four subscales resulted in inconclusive evidence. In confirmatory factor analysis, four subscales were found, and as far as we know current literature does not support the existence of four subscales in the depression construct. Therefore, structural validity was considered poor, with moderate evidence supporting this finding. Reliability, construct validity (hypothesis testing) and criterion validity 


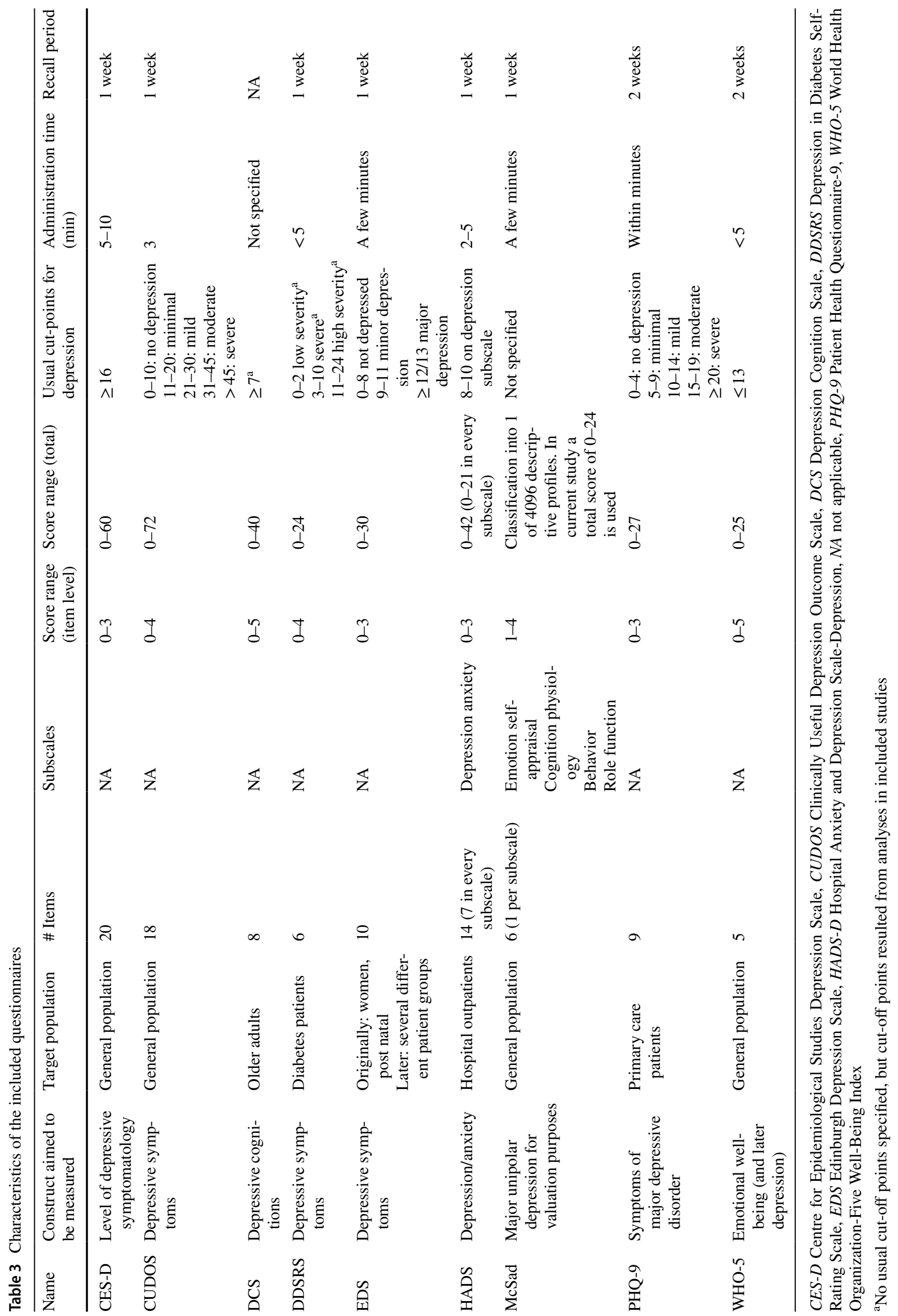


Table 4 Methodological quality of the included studies per measurement property

\begin{tabular}{|c|c|c|c|c|c|c|c|c|}
\hline Publication & $\begin{array}{l}\text { Used question- } \\
\text { naire }\end{array}$ & $\begin{array}{l}\text { Internal con- } \\
\text { sistency }\end{array}$ & Reliability & $\begin{array}{l}\text { Content valid- } \\
\text { ity }\end{array}$ & $\begin{array}{l}\text { Structural } \\
\text { validity }\end{array}$ & $\begin{array}{l}\text { Hypotheses } \\
\text { testing }\end{array}$ & $\begin{array}{l}\text { Cross- } \\
\text { cultural } \\
\text { validity }\end{array}$ & $\begin{array}{l}\text { Criterion } \\
\text { validity }\end{array}$ \\
\hline $\begin{array}{l}\text { Awata et al. } \\
{[23]}\end{array}$ & WHO-5 & Good & & & Good & Fair & Fair & Poor \\
\hline $\begin{array}{l}\text { Carter et al. } \\
\text { [42] }\end{array}$ & CES-D & & & & Good & & & \\
\hline $\begin{array}{l}\text { de Cock et al. } \\
\text { [24] }\end{array}$ & EDS & & & & Good & & & \\
\hline $\begin{array}{l}\text { Hajos et al. } \\
\text { [25] }\end{array}$ & WHO-5 & Excellent & & & Excellent & Good & & Poor \\
\hline Hsu et al. [26] & CUDOS & Good & Good & & Good & Fair & Fair & Fair \\
\hline $\begin{array}{l}\text { Janssen et al. } \\
\text { [43] }\end{array}$ & PHQ-9 & Poor & & & Good & & & Good \\
\hline Kokoszka [27] & DDSRS & Poor & & & & Poor & & Poor \\
\hline $\begin{array}{l}\text { Lamers et al. } \\
\text { [28] }\end{array}$ & PHQ-9 & Poor & Poor & & & Fair & & Good \\
\hline $\begin{array}{l}\text { Lehman et al. } \\
\text { [29] }\end{array}$ & CES-D & Good & & & Good & Good & & \\
\hline $\begin{array}{l}\text { Lloyd et al. } \\
\text { [30] }\end{array}$ & $\begin{array}{l}\text { PHQ-9/WHO- } \\
5\end{array}$ & & & Poor & & & Poor & \\
\hline $\begin{array}{l}\text { Papageorgiou } \\
\text { et al. [31] }\end{array}$ & McSad & & & & & Excellent & & \\
\hline $\begin{array}{l}\text { Rankin et al. } \\
\text { [32] }\end{array}$ & CES-D & Poor & & & & Poor & Fair & \\
\hline $\begin{array}{l}\text { Reddy et al. } \\
\text { [33] }\end{array}$ & $\begin{array}{l}\text { PHQ-9/HADS- } \\
\text { D }\end{array}$ & & Excellent & & Excellent & Good & & \\
\hline $\begin{array}{l}\text { Sousa et al. } \\
{[34]}\end{array}$ & DCS & Fair & & & & & Good & \\
\hline $\begin{array}{l}\text { Sousa et al. } \\
\text { [35] }\end{array}$ & DCS & Good & & & Good & Good & & \\
\hline Stahl et al. [37] & CES-D & Poor & & & & & & Fair \\
\hline $\begin{array}{l}\text { Sultan and } \\
\text { Fisher [36] }\end{array}$ & CES-D & Good & & & Good & Good & & \\
\hline $\begin{array}{l}\text { Zauszniewski } \\
\text { et al. [38] }\end{array}$ & CES-D & Good & & & & Good & & \\
\hline $\begin{array}{l}\text { Zauszniewski } \\
\text { and Graham } \\
\text { [39] }\end{array}$ & DCS & Good & & & & Good & & \\
\hline $\begin{array}{l}\text { Zhang et al. } \\
\text { [40] }\end{array}$ & PHQ-9 & Fair & Poor & & & & & Good \\
\hline $\begin{array}{l}\text { Zhang et al. } \\
\text { [41] }\end{array}$ & CES-D & Poor & Poor & & Good & & & Good \\
\hline
\end{tabular}

$C E S$ - $D$ Centre for Epidemiological Studies Depression Scale, CUDOS Clinically Useful Depression Outcome Scale, DCS Depression Cognition Scale, DDSRS Depression in Diabetes Self-Rating Scale, EDS Edinburgh Depression Scale, HADS-D Hospital Anxiety and Depression ScaleDepression, $P H Q-9$ Patient Health Questionnaire-9, WHO-5 World Health Organization-Five Well-Being Index

were good for the CUDOS-Chinese, with moderate to limited evidence for these findings.

\section{DCS}

The DCS was evaluated in three different studies, using two different languages (English and Portuguese) [34, $35,38]$. There was strong evidence for good internal consistency and structural validity. One study showed moderately strong evidence of good cross-cultural validity of the Portuguese translation of the DCS [34]. Hypothesis testing resulted in inconclusive findings. Reliability, measurement error, content validity, responsiveness and interpretability were not assessed. 


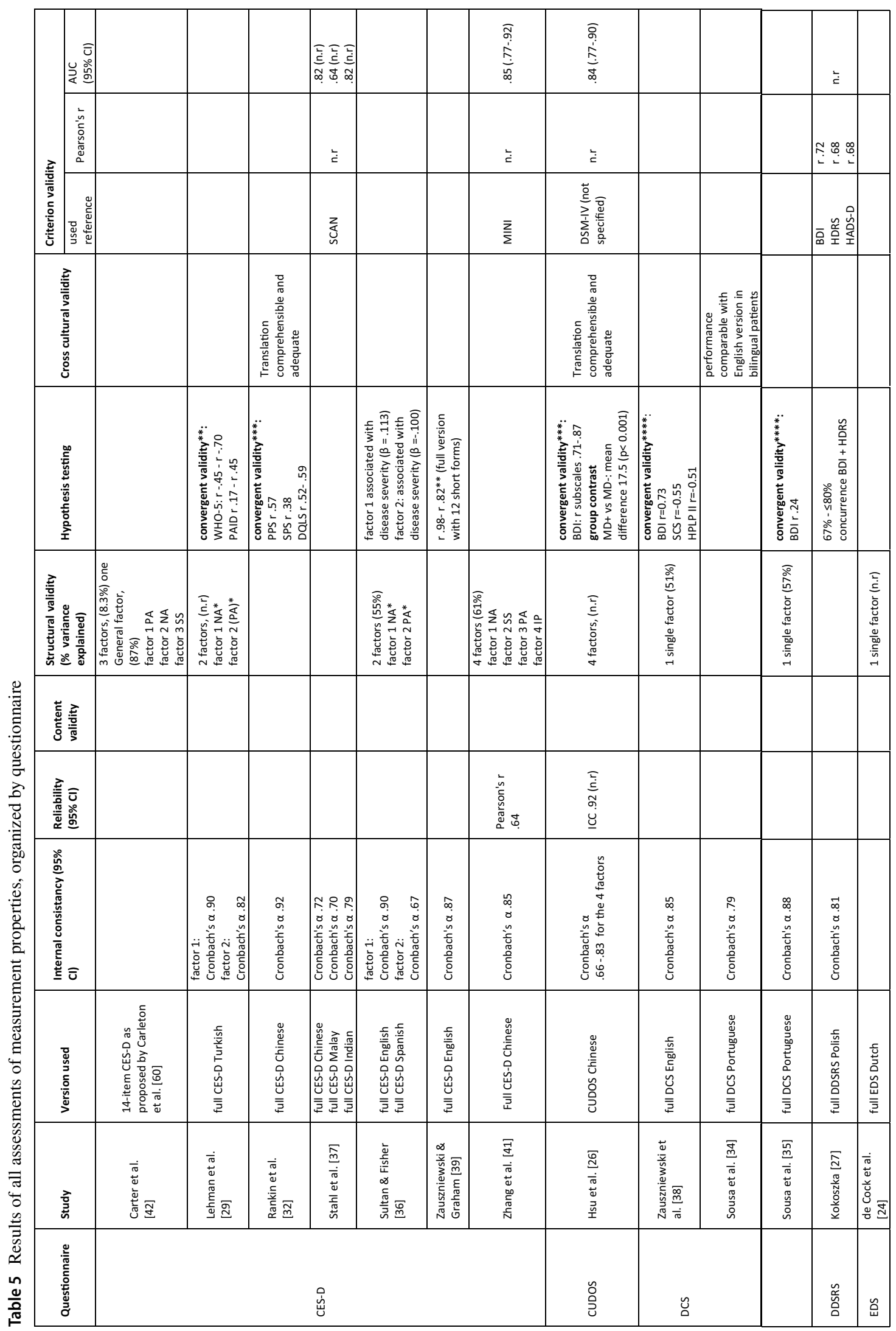




\begin{tabular}{|c|c|c|c|c|c|c|c|c|c|c|}
\hline 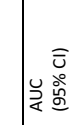 & & & 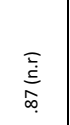 & 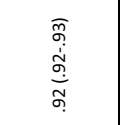 & & & 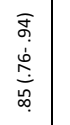 & 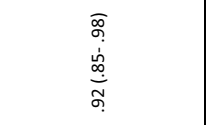 & 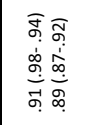 & \\
\hline 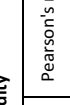 & & & 㞱 & 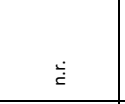 & & & $\grave{c}$ & $\grave{亡}$ & $\grave{\grave{E}}$ & \\
\hline 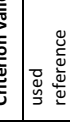 & & & $\overline{\bar{z}}$ & $\overline{\bar{z}}$ & & & $\overline{\bar{z}}$ & $\overline{\bar{O}}$ & 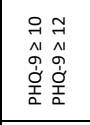 & \\
\hline 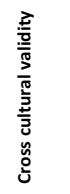 & & & & & 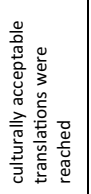 & & & 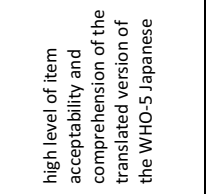 & & 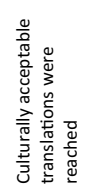 \\
\hline 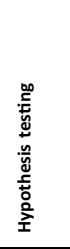 & 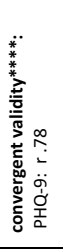 & 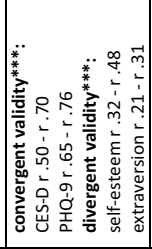 & & 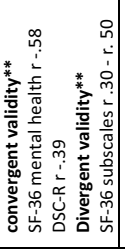 & & 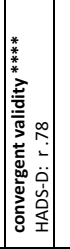 & & 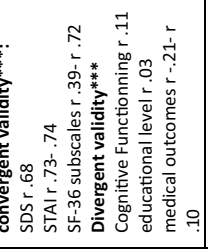 & 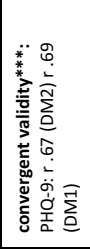 & \\
\hline 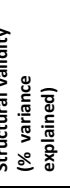 & 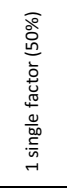 & & 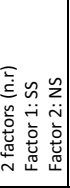 & & & 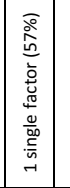 & & 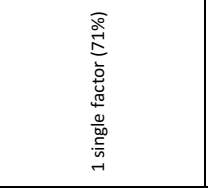 & 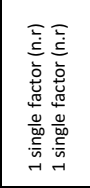 & \\
\hline 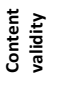 & & & & & 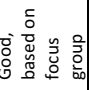 & & & & & 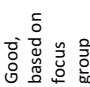 \\
\hline 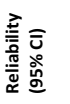 & & & & 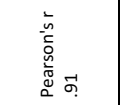 & & & 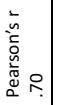 & & & \\
\hline 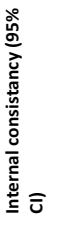 & 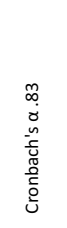 & & 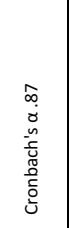 & 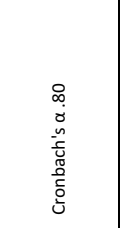 & & 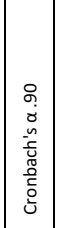 & 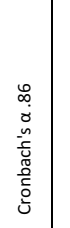 & 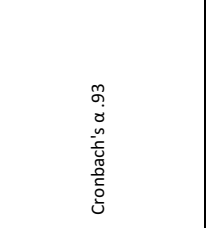 & 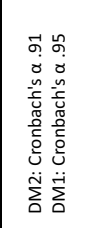 & \\
\hline 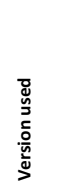 & 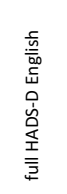 & 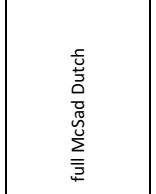 & 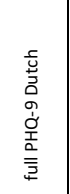 & 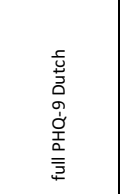 & 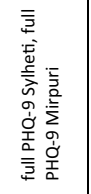 & 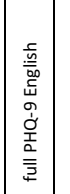 & 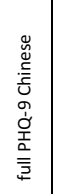 & 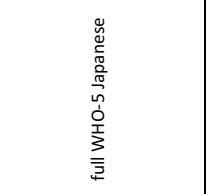 & 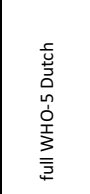 & 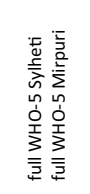 \\
\hline 旁 & 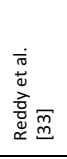 & 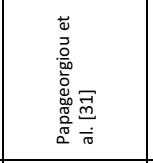 & 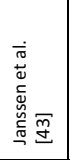 & 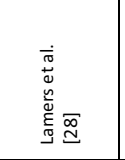 & 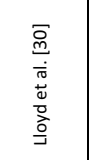 & 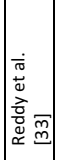 & 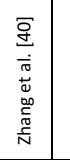 & 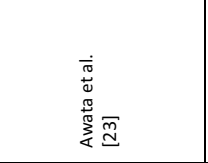 & 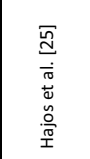 & 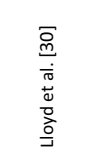 \\
\hline 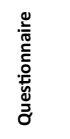 & $\begin{array}{l}\text { 㞻 } \\
\text { 岸 }\end{array}$ & $\begin{array}{l}\overline{\mathscr{J}} \\
\stackrel{\widetilde{g}}{\Sigma}\end{array}$ & \multicolumn{5}{|c|}{$\begin{array}{l}\text { oे } \\
\text { 옴 }\end{array}$} & \\
\hline
\end{tabular}

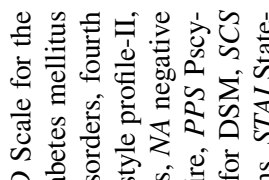

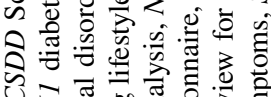

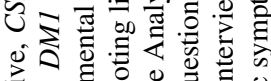

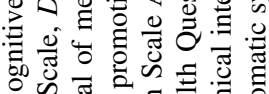

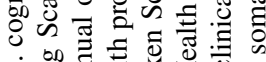

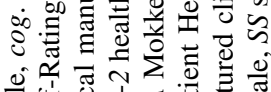

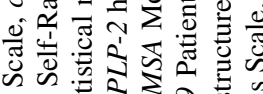

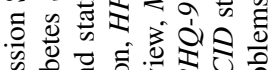

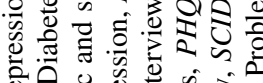

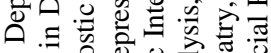
品

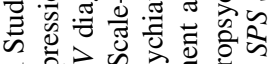

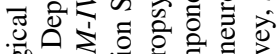

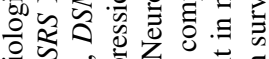

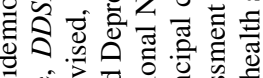

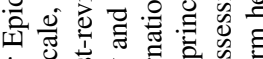

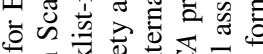

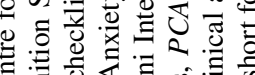

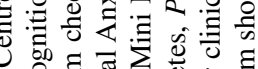
U ن

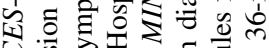

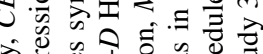

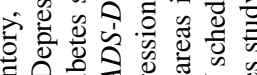

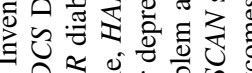

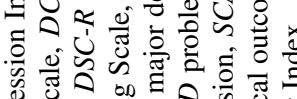

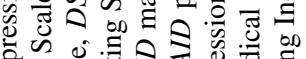

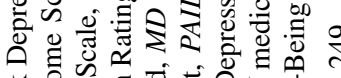

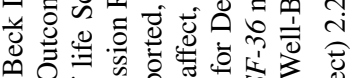

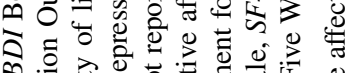

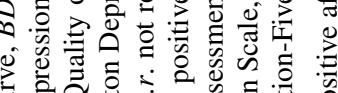

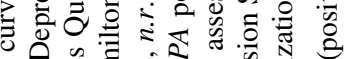

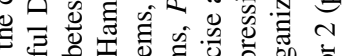

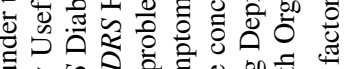

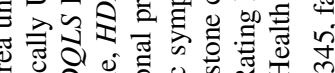

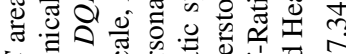

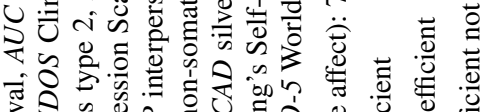

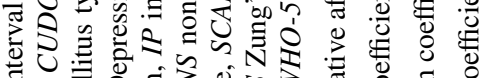

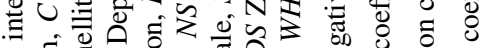

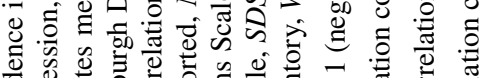

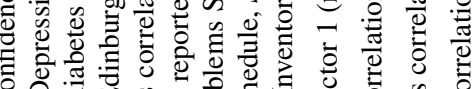

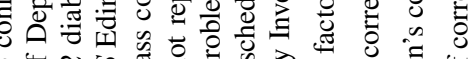

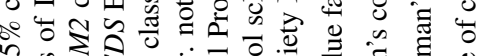

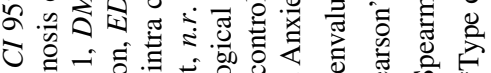

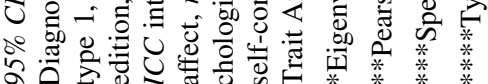




\section{EDS}

Only one study [24] assessed a Dutch version of the EDS. Within this study only structural validity was assessed. Since this was done with good methodological quality and the analysis yielded one single, theoretically explicable factor, evidence regarding structural validity was considered moderate for good structural validity.

\section{HADS-D}

One single study [33] assessed measurement properties of the HADS-D. There was strong evidence for good internal consistency and structural validity and moderate evidence for good construct validity (hypothesis testing).

\section{McSad}

The construct validity of the Dutch McSad was evaluated in one study using hypothesis testing [31]. The methodological quality of this assessment was rated excellent, resulting in confirmation of all pre-set hypothesis. The level of evidence was therefore rated 'strong' for good construct validity.

\section{PHQ-9}

Measurement properties of the Patient Health Questionnaire (PHQ) were assessed in five different studies in five different languages [28, 30, 33, 40, 43]. Reliability, content validity, cross-cultural validity, internal consistency, structural validity and criterion validity were assessed. However, assessments of reliability, content validity and cross-cultural validity were not included in the data synthesis, since these were of poor methodological quality. There was strong evidence of good internal consistency and criterion validity. Construct validity (hypothesis testing) was rated 'good' with a moderate level of evidence. The evidence for structural validity was inconclusive, since two studies of at least good quality found different factor structures $[33,43]$.

\section{WHO-5}

Measurement properties of the WHO-5 were assessed by three different studies in four different languages [23, 25, 30]. Reliability, measurement error, responsiveness and interpretability were not assessed and the assessments of content validity and criterion validity were of poor methodological quality. Internal consistency was good, with strong level of evidence. Evidence for good structural validity and construct validity (hypothesis testing) was moderate. There was limited evidence for good cross-cultural validity of the WHO-5 [23].

\section{Discussion}

We identified 21 studies evaluating the measurement properties of nine different questionnaires for measuring depressive symptoms in diabetes patients. Overall, the CES-D performed best, with strong evidence for a positive internal consistency, structural validity, and construct validity, moderate evidence for a positive criterion validity and limited evidence for positive cross-cultural validity. Insufficient information was available on content validity and reliability.

The use of the WHO-5 was supported by strong evidence for a positive internal consistency and moderate evidence for a positive structural validity and construct validity. However, the WHO-5 is originally developed as a questionnaire to measure the level of emotional well-being and not to assess depressive symptoms. Yet, caution should be applied when choosing the WHO-5 to specifically measure depressive symptoms. The PHQ-9 is frequently studied amongst patients with diabetes. We found strong evidence for a positive internal consistency and positive criterion validity and moderate evidence for positive construct validity. However, since the evidence for its structural validity is inconclusive, caution should be applied when the PHQ-9 is used for evaluative purposes. For all other questionnaires, evidence is too limited to draw any definitive conclusions regarding their measurement properties. Therefore, based on the current evidence, we recommend using the CES-D for evaluating depressive symptoms in patients with diabetes. However, for none of the questionnaires complete information is available on all measurement properties when used in a population of adults with diabetes. One important shortcoming is lack of evidence on the content validity of the questionnaires, including the CES-D, in diabetes patients. Content validity is often considered the most important measurement property because it can affect all other measurement properties. Therefore, we recommend further literature review on the content validity of these questionnaires in other populations, as well as qualitative studies with patients and professionals on the relevance, comprehensiveness, and comprehensibility of these questionnaires in diabetes patients. Furthermore, measurement error, responsiveness and interpretability were not assessed for any of the questionnaires. This is important since shortcomings in any of the measurement properties pose a considerable threat to the ability of a questionnaire to measure depression in diabetes patients [50].

Our systematic review adds to the current literature by providing a structured and comprehensive overview of the measurement properties of depression questionnaires used in diabetes patients and the methodological quality of the 
Table 6 Levels of evidence for the quality of the questionnaires

\begin{tabular}{llllllll}
\hline & $\begin{array}{l}\text { Internal } \\
\text { consistency }\end{array}$ & Reliability & $\begin{array}{l}\text { Content } \\
\text { validity }\end{array}$ & $\begin{array}{l}\text { Structural } \\
\text { validity }\end{array}$ & $\begin{array}{l}\text { Hypothe- } \\
\text { sis testing }\end{array}$ & $\begin{array}{l}\text { Cross-cul- } \\
\text { tural validity }\end{array}$ & Criterion validity \\
\hline CES-D & +++ & NA & NA & +++ & +++ & + & ++ \\
CUDOS & ++ & ++ & NA & -- & + & + & + \\
DCS & +++ & NA & NA & +++ & $+l$ & ++ & NA \\
DDSRS & $?$ & NA & NA & NA & $?$ & NA & $?$ \\
EDS & NA & NA & NA & ++ & NA & NA & NA \\
HADS & +++ & NA & NA & +++ & ++ & NA & NA \\
McSad & NA & NA & NA & NA & +++ & NA & NA \\
PHQ-9 & +++ & $?$ & $?$ & $+l-$ & ++ & $?$ & +++ \\
WHO-5 & +++ & $?$ & $?$ & ++ & ++ & + & $?$ \\
\hline
\end{tabular}

+++ strong positive evidence +++ moderate positive evidence; + limited positive evidence; --- strong negative evidence; - - moderate negative evidence; +/- inconclusive; ? unknown, due to poor methodological quality; $N A$ no information available studies assessing them. Also, this review provides recommendations on their use. By describing which questionnaires are-at this point in time-best supported by the evidence, this review is of use when choosing a questionnaire to monitor depression in daily practice. Previously, Roy et al. conducted a comprehensive review of depression screening questionnaires and their operating characteristics in diabetes populations [16]. In their review, 23 relevant studies were identified. There is only limited overlap in studies $(n=5)$ between the study of Roy et al., and our review. This is mainly because we included studies that assessed measurement properties of questionnaires used to evaluate depressive symptoms (for evaluative purposes), and we excluded studies assessing diagnostic test accuracy of questionnaires used for screening or detecting a depressive disorder. Roy et al. concluded that there is lack of evidence on the reliability and validity of depression questionnaires used for patients with diabetes to provide recommendations. In our more recent and up-to-date review, more evidence was available to provide recommendations for measuring change in depressive symptoms.

Other reviews assessing the use of depression questionnaires in patients with chronic medical illnesses (for example, in patients with cancer [51] and Parkinson's disease [52]) provided comparable recommendations, suggesting that our findings are robust. However, we are aware that we need to be cautious in recommending the use of the CES-D because of the lack of evidence on some important measurement properties, like reliability and responsiveness.

A new development in measuring and monitoring patient-reported health is the use of item banks based on Item Response Theory (IRT), such as those from the Patient Reported Outcomes Measurement Information System (PROMIS) (http://www.healthmeasures.net/promis). IRTbased item banks enable Computer Adaptive Testing (CAT), in which, after a starting question, the computer selects subsequent questions based on the answers to previous questions. With CAT patients need to complete on average only 5-9 questions to get a reliable score, which makes this method a highly efficient and patient-friendly way of measuring. The PROMIS Depression instruments seem to be valid and reliable for measuring depressive symptoms [53, 54]. Recent studies indicate that the PROMIS Depression CAT can be more easily used in clinical practice than the CES-D and PHQ-9 since it can be adapted to the needs in a specific care setting, while it results in comparable scores [55-57]. The PROMIS methodology is promising for use in patients with a chronic physical illness, minimizing the impact of somatic symptoms on depression scores while retaining enough uniformity to compare between patient populations and other depression measures [57]. Therefore, in time, PROMIS might replace the traditional depression questionnaires.

This study is the first to systematically summarize the evidence on the measurement properties of questionnaires measuring depressive symptoms in patients with diabetes. A strength of this study is the use of the standardized COSMIN methodology for critical appraisal of the methodological quality of these studies, the quality of the questionnaires and the level of evidence. Another strength of this review is its inclusive search strategy, thereby limiting chances of missing important studies.

The following two limitations apply. Firstly, the identified depression questionnaires were assessed in a large variety of languages and settings, but whether the results on the individual questionnaires discussed in this review can be validly generalized across language versions is not clear. Only few identified studies performed a cross-cultural validation of translated questionnaires in a diabetes population. Questionnaires may perform differently across different languages and cultures [21].

Secondly, to provide a comprehensive overview of measurement properties of depression questionnaires in diabetes patients, we deliberately excluded studies that only assessed 
diagnostic accuracy. Although this distinction is based on a theoretically sound concept and rests on differences in the use and purpose of a questionnaire (monitoring vs. diagnosing), in the various studies, this distinction was often not clearly made. This resulted in some difficulties deciding whether or not a study should be included in the review.

Further studies are needed on the measurement properties of depression questionnaires in diabetes patients. The finding that internal consistency, hypothesis testing and structural validity are the most evaluated properties is in line with other literature [22, 58, 59]. However, not all measurement properties (measurement error, responsiveness and interpretability) are extensively evaluated and further research is needed to provide definitive recommendations.

In summary, this systematic review constitutes an important knowledge base for health care providers and researchers by providing a comprehensive overview of questionnaires measuring depressive symptoms in diabetes patients. The CES-D has the strongest evidence for good measurement properties for measuring depressive symptoms in patients with diabetes.

\section{Compliance with ethical standards}

Conflict of interest CT is one of the founders of the COSMIN initiative. The authors report no other competing interests.

Open Access This article is distributed under the terms of the Creative Commons Attribution 4.0 International License (http://creativeco mmons.org/licenses/by/4.0/), which permits unrestricted use, distribution, and reproduction in any medium, provided you give appropriate credit to the original author(s) and the source, provide a link to the Creative Commons license, and indicate if changes were made.

\section{References}

1. Guariguata, L., Whiting, D., Hambleton, I., Beagley, J., Linnenkamp, U., \& Shaw, J. (2014). Global estimates of diabetes prevalence for 2013 and projections for 2035. Diabetes Research and Clinical Practice, 103(2), 137-149.

2. Anderson, R. J., Freedland, K. E., Clouse, R. E., \& Lustman, P. J. (2001). The prevalence of comorbid depression in adults with diabetes: A meta-analysis. Diabetes Care, 24(6), 1069-1078.

3. Roy, T., \& Lloyd, C. E. (2012). Epidemiology of depression and diabetes: A systematic review. Journal of Affective Disorders, 142, S8-S21. https://doi.org/10.1016/S0165-0327(12)70004-6.

4. Bot, M., Pouwer, F., Ormel, J., Slaets, J. P., \& De Jonge, P. (2010). Predictors of incident major depression in diabetic outpatients with subthreshold depression. Diabetic Medicine, 27(11), 1295-1301.

5. Gonzalez, J. S., Peyrot, M., McCarl, L. A., Collins, E. M., Serpa, L., Mimiaga, M. J., et al. (2008). Depression and diabetes treatment nonadherence: A meta-analysis. Diabetes Care, 31(12), 2398-2403.

6. Lustman, P. J., \& Clouse, R. E. (2005). Depression in diabetic patients. Journal of Diabetes and Its Complications, 19(2), 113122. https://doi.org/10.1016/j.jdiacomp.2004.01.002.
7. Lin, E. H., Rutter, C. M., Katon, W., Heckbert, S. R., Ciechanowski, P., Oliver, M. M., et al. (2010). Depression and advanced complications of diabetes: A prospective cohort study. Diabetes Care, 33(2), 264-269. https://doi.org/10.2337/dc09-1068.

8. Ali, S., Stone, M., Skinner, T. C., Robertson, N., Davies, M., \& Khunti, K. (2010). The association between depression and health-related quality of life in people with type 2 diabetes: A systematic literature review. Diabetes/Metabolism Research and Reviews, 26(2), 75-89. https://doi.org/10.1002/dmrr.1065.

9. Bosmans, J. E., \& Adriaanse, M. C. (2012). Outpatient costs in pharmaceutically treated diabetes patients with and without a diagnosis of depression in a Dutch primary care setting. BMC Health Services Research, 12, 46. https://doi. org/10.1186/1472-6963-12-46.

10. Association., A. D. (2013). Standards of medical care in diabetes-2013. Diabetes Care, 36, S11.

11. Katon, W., Unützer, J., Wells, K., \& Jones, L. (2010). Collaborative depression care: History, evolution and ways to enhance dissemination and sustainability. General Hospital Psychiatry, 32(5), 456-464.

12. Radloff, L. S. (1977). The CES-D scale a Self-Report Depression Scale for research in the general population. Applied Psychological Measurement, 1(3), 385-401.

13. Zigmond, A. S., \& Snaith, R. P. (1983). The hospital Anxiety and Depression Scale. Acta Psychiatrica Scandinavica, 67(6), 361-370.

14. Kroenke, K., \& Spitzer, R. L. (2002). The PHQ-9: A new depression diagnostic and severity measure. Psychiatric Annals, 32(9), $1-7$.

15. Beck, A. T., Steer, R. A., \& Brown, G. K. (1996). Manual for the Beck Depression Inventory-II. San Antonio, TX: Psychological Corporation.

16. Roy, T., Lloyd, C. E., Pouwer, F., Holt, R. I., \& Sartorius, N. (2012). Screening tools used for measuring depression among people with type 1 and type 2 diabetes: A systematic review. Diabetic Medicine, 29(2), 164-175. https://doi.org/10.111 1/j.1464-5491.2011.03401.x.

17. Mokkink, L. B., Terwee, C. B., Patrick, D. L., Alonso, J., Stratford, P. W., Knol, D. L., et al. (2010). The COSMIN study reached international consensus on taxonomy, terminology, and definitions of measurement properties for health-related patient-reported outcomes. Journal of Clinical Epidemiology, 63(7), 737-745.

18. Mokkink, L. B., Terwee, C. B., Patrick, D. L., Alonso, J., Stratford, P. W., Knol, D. L., et al. (2010). The COSMIN checklist for assessing the methodological quality of studies on measurement properties of health status measurement instruments: An international Delphi study. Qual Life Research, 19(4), 539-549. https:// doi.org/10.1007/s11136-010-9606-8.

19. Terwee, C. B., Mokkink, L. B., Knol, D. L., Ostelo, R. W., Bouter, L. M., \& de Vet, H. C. (2012). Rating the methodological quality in systematic reviews of studies on measurement properties: A scoring system for the COSMIN checklist. Qual Life Research. https://doi.org/10.1007/s11136-011-9960-1.

20. Terwee, C. B., Bot, S. D., de Boer, M. R., van der Windt, D. A., Knol, D. L., Dekker, J., et al. (2007). Quality criteria were proposed for measurement properties of health status questionnaires. J Clinical Epidemiology, 60(1), 34-42. https://doi.org/10.1016/j. jclinepi.2006.03.012.

21. Schellingerhout, J. M., Heymans, M. W., Verhagen, A. P., de Vet, H. C., Koes, B. W., \& Terwee, C. B. (2011). Measurement properties of translated versions of neck-specific questionnaires: A systematic review. BMC Medical Research Methodology, 11(1), 87.

22. Schellingerhout, J. M., Verhagen, A. P., Heymans, M. W., Koes, B. W., Henrica, C., \& Terwee, C. B. (2012). Measurement properties 
of disease-specific questionnaires in patients with neck pain: A systematic review. Quality of Life Research, 21(4), 659-670.

23. Awata, S., Bech, P., Yoshida, S., Hirai, M., Suzuki, S., Yamashita, M., et al. (2007). Reliability and validity of the Japanese version of the World Health Organization-Five Well-Being Index in the context of detecting depression in diabetic patients. Psychiatry and Clinical Neurosciences, 61(1), 112-119.

24. de Cock, E. S., Emons, W. H., Nefs, G., Pop, V. J., \& Pouwer, F. (2011). Dimensionality and scale properties of the Edinburgh Depression Scale (EDS) in patients with type 2 diabetes mellitus: The DiaDDzoB study. BMC Psychiatry, 11, 141. https://doi. org/10.1186/1471-244x-11-141.

25. Hajos, T. R., Pouwer, F., Skovlund, S. E., Den Oudsten, B. L., Geelhoed-Duijvestijn, P. H., Tack, C. J., et al. (2013). Psychometric and screening properties of the WHO-5 well-being index in adult outpatients with type 1 or type 2 diabetes mellitus. Diabetic Medicine, 30(2), e63-e69. https://doi.org/10.1111/ dme. 12040 .

26. Hsu, L. F., Kao, C. C., Wang, M. Y., Chang, C. J., \& Tsai, P. S. (2014). Psychometric testing of a Mandarin Chinese version of the clinically useful depression outcome scale for patients diagnosed with type 2 diabetes mellitus. International Journal of Nursing Studies, 51(12), 1595-1604. https://doi.org/10.1016/j. ijnurstu.2014.05.004.

27. Kokoszka, A. (2008). Depression in diabetes self-rating scale: A screening tool. Diabetologia Doswiadczalna i Kliniczna, 8(1), 43-47. Retrieved from http://www.ddk.viamedica.pl.

28. Lamers, F., Jonkers, C. C., Bosma, H., Penninx, B. W., Knottnerus, J. A., \& van Eijk, J. T. (2008). Summed score of the Patient Health Questionnaire-9 was a reliable and valid method for depression screening in chronically ill elderly patients. Journal of Clinical Epidemiology, 61(7), 679-687. https://doi.org/10.1016/j.jclin epi.2007.07.018

29. Lehmann, V., Makine, C., Karşıdağ, Ç, Kadıŏ̆lu, P., Karşıdă̆, K., \& Pouwer, F. (2011). Validation of the Turkish version of the Centre for Epidemiologic Studies Depression Scale (CES-D) in patients with type 2 diabetes mellitus. BMC Medical Research Methodology, 11(1), 109.

30. Lloyd, C. E., Roy, T., Begum, S., Mughal, S., \& Barnett, A. H. (2012). Measuring psychological well-being in South Asians with diabetes; a qualitative investigation of the PHQ-9 and the WHO-5 as potential screening tools for measuring symptoms of depression. Diabetic Medicine, 29(1), 140-147. https://doi.org/10.111 1/j.1464-5491.2011.03481.x.

31. Papageorgiou, K., Vermeulen, K. M., Schroevers, M. J., Buskens, E., \& Ranchor, A. V. (2013). Testing the McSad depression specific classification system in patients with somatic conditions: Validity and performance. Health Qual Life Outcomes, 11, 125. https://doi.org/10.1186/1477-7525-11-125.

32. Rankin, S. H., Galbraith, M. E., \& Johnson, S. (1993). Reliability and validity data for a Chinese translation of the center for epidemiological studies-depression. Psychological Reports, 73(3 Pt 2), 1291-1298. https://doi.org/10.2466/pr0.1993.73.3f.1291.

33. Reddy, P., Philpot, B., Ford, D., \& Dunbar, J. A. (2010). Identification of depression in diabetes: The efficacy of PHQ-9 and HADSD. British Journal of General Practice, 60(575), e239-e245.

34. Sousa, V. D., Zauszniewski, J. A., Mendes, I. A., \& Zanetti, M. L. (2005). Cross-cultural equivalence and psychometric properties of the Portuguese version of the Depressive Cognition Scale. Journal of Nursing Measurement, 13(2), 87-99.

35. Sousa, V. D., Zanetti, M. L., Zauszniewski, J. A., Mendes, I. A., \& Daguano, M. O. (2008). Psychometric properties of the Portuguese version of the Depressive Cognition Scale in Brazilian adults with diabetes mellitus. Journal of Nursing Measurement, 16(2), 125-135.
36. Sultan, S., \& Fisher, L. (2010). Depression as a proxy of negative affect? A critical examination of the use of the CES-D in type 2 diabetes. Revue Européenne de Psychologie Appliquée/European Review of Applied Psychology, 60(2), 97-104.

37. Stahl, D., Sum, C. F., Lum, S. S., Liow, P. H., Chan, Y. H., Verma, S., et al. (2008). Screening for depressive symptoms: Validation of the Center for Epidemiologic Studies Depression Scale (CESD) in a multiethnic group of patients with diabetes in Singapore. Diabetes Care, 31(6), 1118-1119.

38. Zauszniewski, J. A., Chung, C., Krafcik, K., \& Sousa, V. D. (2001). Psychometric testing of the depressive cognition scale in women with type 2 diabetes. Journal of Nursing Measurement, 9(1), 61-72.

39. Zauszniewski, J. A., \& Graham, G. C. (2009). Comparison of short scales to measure depressive symptoms in elders with diabetes. Western Journal of Nursing Research, 31(2), 219-234.

40. Zhang, Y., Ting, R., Lam, M., Lam, J., Nan, H., Yeung, R., et al. (2013). Measuring depressive symptoms using the Patient Health Questionnaire-9 in Hong Kong Chinese subjects with type 2 diabetes. Journal of Affective Disorders, 151(2), 660-666. https:// doi.org/10.1016/j.jad.2013.07.014.

41. Zhang, Y., Ting, R. Z., Lam, M. H., Lam, S. P., Yeung, R. O., Nan, H., et al. (2015). Measuring depression with CES-D in Chinese patients with type 2 diabetes: The validity and its comparison to PHQ-9. BMC Psychiatry, 15, 198. https://doi.org/10.1186/s1288 8-015-0580-0.

42. Carter, J., Cogo-Moreira, H., Herrmann, N., Merino, D., Yang, P., Shah, B. R., et al. (2016). Validity of the Center for Epidemiological Studies Depression Scale in type 2 diabetes. Journal of Psychosomatic Research, 90, 91-97.

43. Janssen, E. P., Köhler, S., Stehouwer, C. D., Schaper, N. C., Dagnelie, P. C., Sep, S. J., et al. (2016). The Patient Health Questionnaire-9 as a screening tool for depression in individuals with type 2 diabetes mellitus: The Maastricht Study. Journal of the American Geriatrics Society, 64(11), e201-e206.

44. Zimmerman, M., Chelminski, I., McGlinchey, J. B., \& Posternak, M. A. (2008). A clinically useful depression outcome scale. Comprehensive Psychiatry, 49(2), 131-140.

45. Zauszniewski, J. A. (1995). Development and testing of a measure of depressive cognitions in older adults. Journal of Nursing Measurement, 3(1), 31-41.

46. Cox, J. L., Holden, J. M., \& Sagovsky, R. (1987). Detection of postnatal depression. Development of the 10-item Edinburgh Postnatal Depression Scale. The British Journal of Psychiatry, 150(6), 782-786.

47. Bennett, K., Torrance, G., Boyle, M., Guscott, R., \& Moran, L. (2000). Development and testing of a utility measure for major, unipolar depression (McSad). Quality of Life Research, 9(1), 109-120.

48. Heun, R., Bonsignore, M., Barkow, K., \& Jessen, F. (2001). Validity of the five-item WHO Well-Being Index (WHO-5) in an elderly population. European archives of Psychiatry and Clinical Neuroscience, 251(2), 27-31.

49. Whiting, P., Rutjes, A. W., Reitsma, J. B., Bossuyt, P. M., \& Kleijnen, J. (2003). The development of QUADAS: A tool for the quality assessment of studies of diagnostic accuracy included in systematic reviews. BMC Medical Research Methodology, 3(1), 25.

50. Mokkink, L. B., Terwee, C. B., Knol, D. L., Stratford, P. W., Alonso, J., Patrick, D. L., et al. (2010). The COSMIN checklist for evaluating the methodological quality of studies on measurement properties: A clarification of its content. BMC Medical Research Methodology, 10, 22. https://doi.org/10.1186/1471-2288-10-22.

51. Luckett, T., Butow, P. N., King, M. T., Oguchi, M., Heading, G., Hackl, N. A., et al. (2010). A review and recommendations for 
optimal outcome measures of anxiety, depression and general distress in studies evaluating psychosocial interventions for Englishspeaking adults with heterogeneous cancer diagnoses. Support Care Cancer, 18(10), 1241-1262. https://doi.org/10.1007/s0052 0-010-0932-8.

52. Torbey, E., Pachana, N. A., \& Dissanayaka, N. N. (2015). Depression rating scales in Parkinson's disease: A critical review updating recent literature. Journal of Affective Disorders, 184, 216-224. https://doi.org/10.1016/j.jad.2015.05.059.

53. Vilagut, G., Forero, C., Adroher, N., Olariu, E., Cella, D., Alonso, J., et al. (2015). Testing the PROMIS® Depression measures for monitoring depression in a clinical sample outside the US. Journal of Psychiatric Research, 68, 140-150.

54. Pilkonis, P. A., Yu, L., Dodds, N. E., Johnston, K. L., Maihoefer, C. C., \& Lawrence, S. M. (2014). Validation of the depression item bank from the Patient-Reported Outcomes Measurement Information System (PROMIS) in a three-month observational study. Journal of Psychiatric Research, 56, 112-119. https://doi. org/10.1016/j.jpsychires.2014.05.010.

55. Kim, J., Chung, H., Askew, R. L., Park, R., Jones, S. M., Cook, K. F., et al. (2015). Translating CESD-20 and PHQ-9 scores to PROMIS depression. Assessment. https://doi.org/10.1177/10731 91115607042.

56. Choi, S. W., Schalet, B., Cook, K. F., \& Cella, D. (2014). Establishing a common metric for depressive symptoms: Linking the
BDI-II, CES-D, and PHQ-9 to PROMIS depression. Psychological Assessment, 26(2), 513-527. https://doi.org/10.1037/a0035 768.

57. Amtmann, D., Kim, J., Chung, H., Bamer, A. M., Askew, R. L., Wu, S., et al. (2014). Comparing CESD-10, PHQ-9, and PROMIS depression instruments in individuals with multiple sclerosis. Rehabilitation Psychology, 59(2), 220-229. https://doi. org/10.1037/a0035919.

58. Wong, C. K., Chen, J., Yu, C. L., Sham, M., \& Lam, C. L. (2015). Systematic review recommends the European organization for research and treatment of cancer colorectal cancer-specific module for measuring quality of life in colorectal cancer patients. $J$ Clinical Epidemiology, 68(3), 266-278. https://doi.org/10.1016/j. jclinepi.2014.09.021.

59. Wigham, S., \& McConachie, H. (2014). Systematic review of the properties of tools used to measure outcomes in anxiety intervention studies for children with autism spectrum disorders. PLoS ONE, 9(1), e85268. https://doi.org/10.1371/journal.pone.00852 68.

60. Carleton R. N., Thibodeau M. A., Teale M. J., Welch P. G., Abrams M. P., Robinson T., \& Asmundson G. J. (2013). The center for epidemiologic studies depression scale: a review with theoretical and empirical examination of item content and factor structure. PLoS ONE, 8(3), e58067. https://doi.org/10.1371/journ al.pone. 0058067 . 